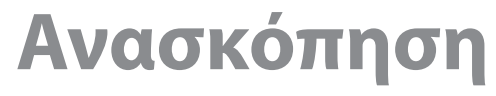 Review
}

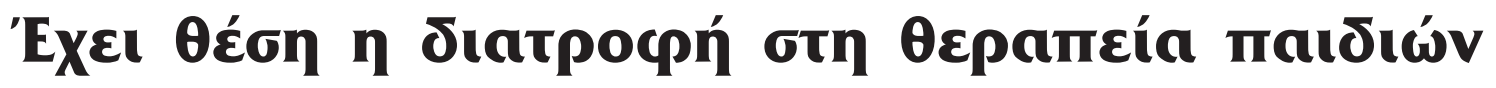

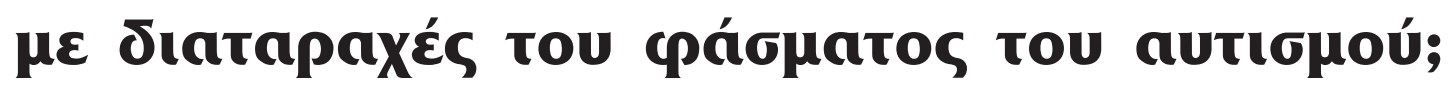

\author{
M. Гஸ́үou, ${ }^{1}$ Г. Koגıó $\varsigma^{2}$

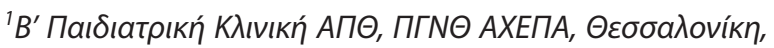

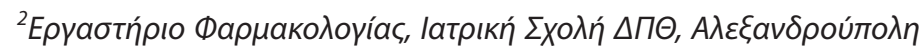 \\ Uuxıатрıкń 2020, 31:57-69
}

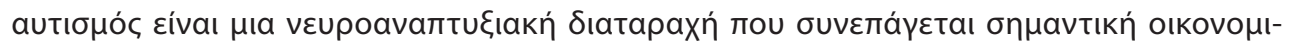

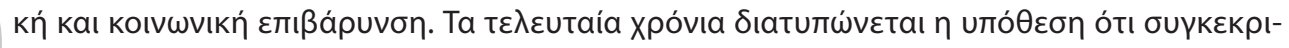

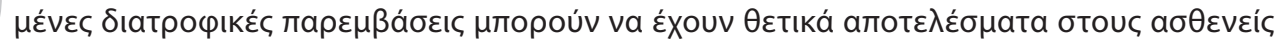

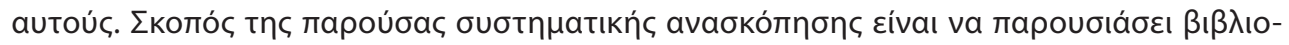

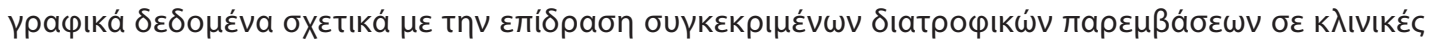

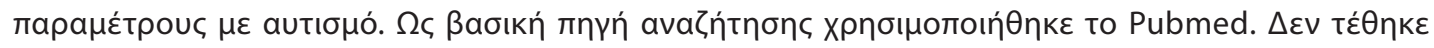

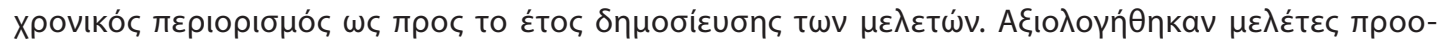

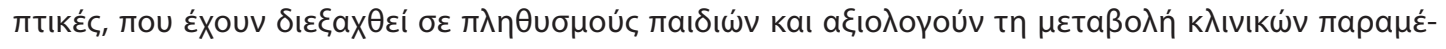

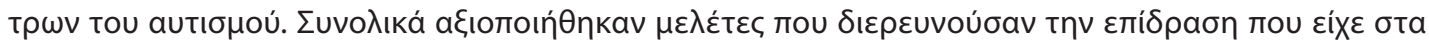

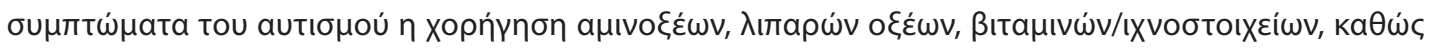

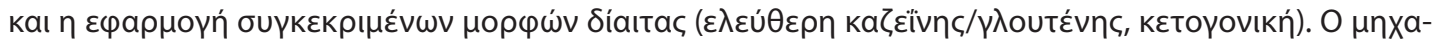

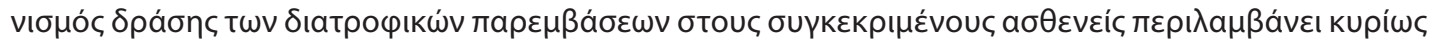

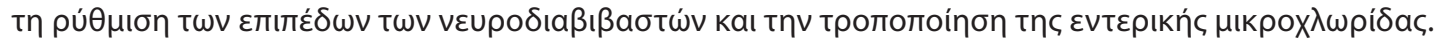

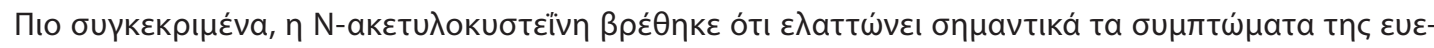

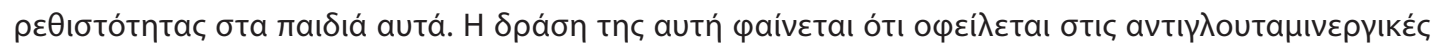

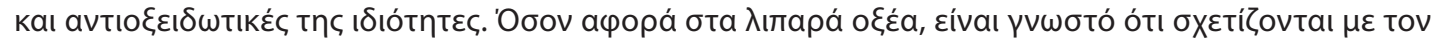

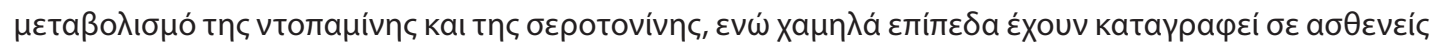

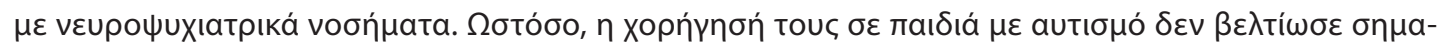

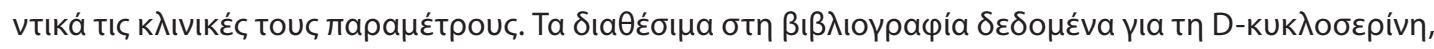

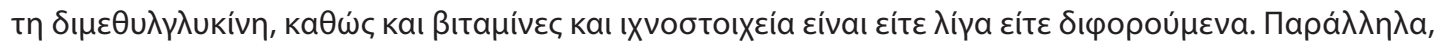

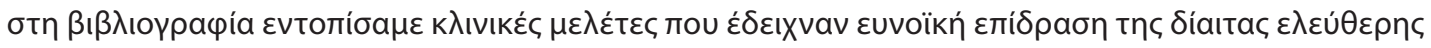

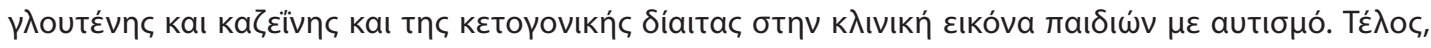




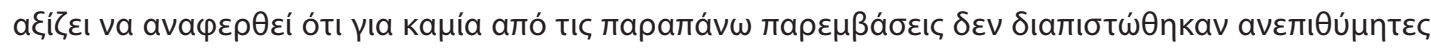

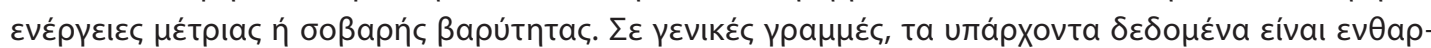

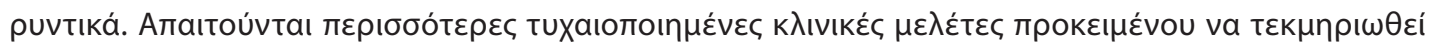

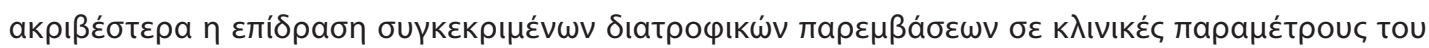
autiouoú.

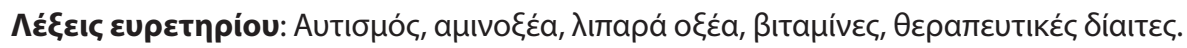

\section{Eıбaywyń}

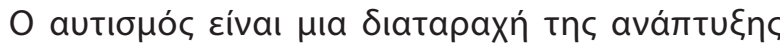

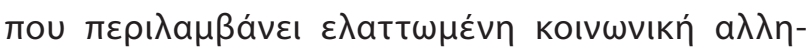

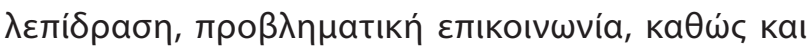

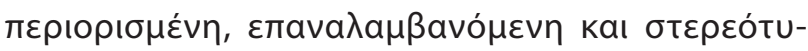

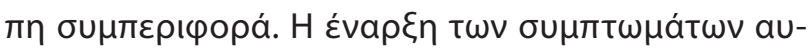

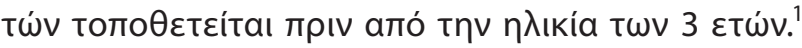

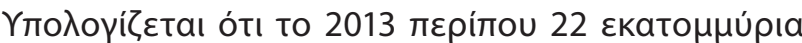

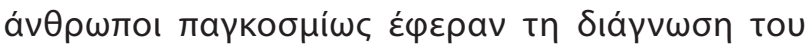

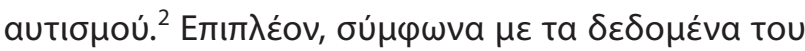

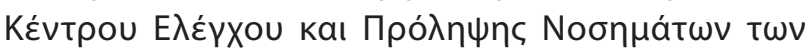

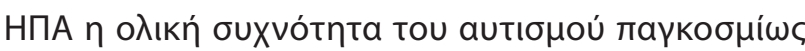

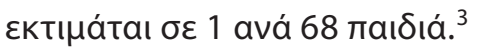

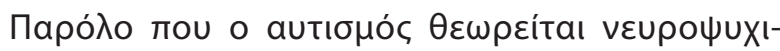

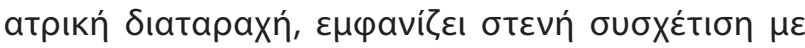

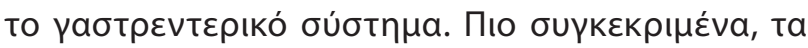

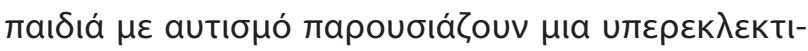

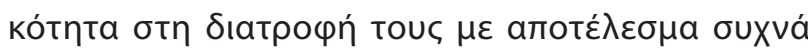

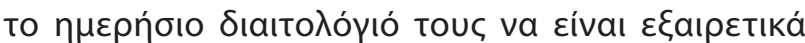

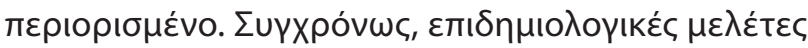

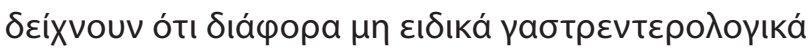

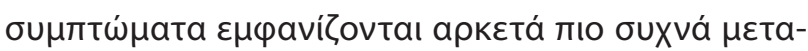

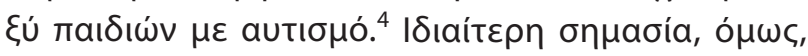

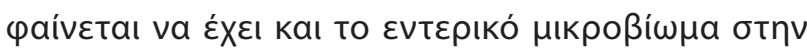

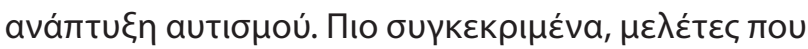

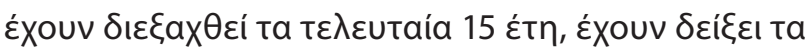

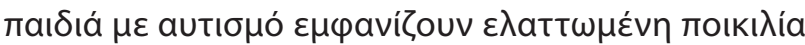

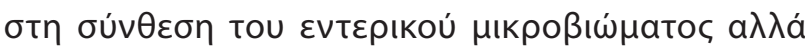

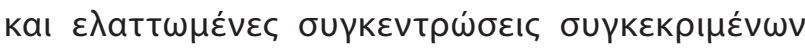

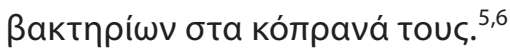

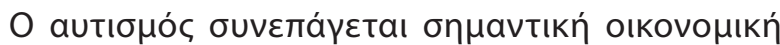

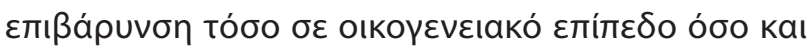

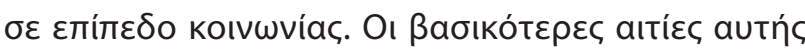

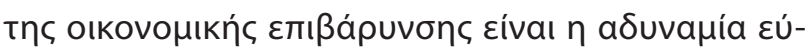

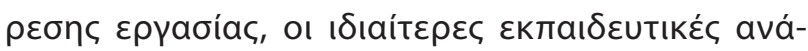

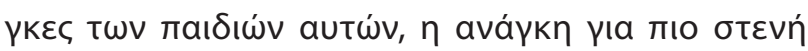

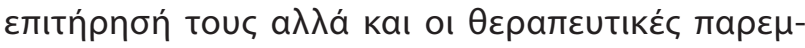

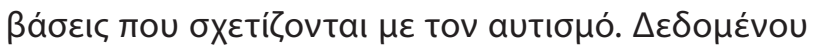

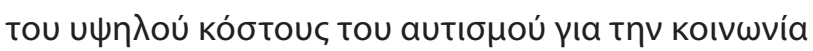

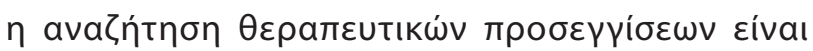

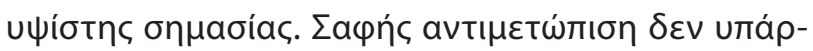

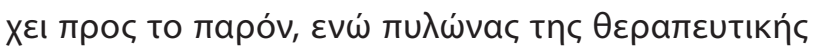

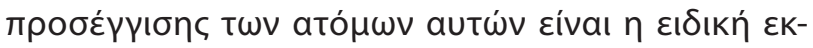

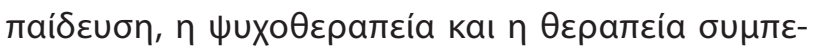

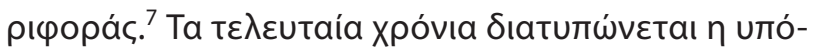

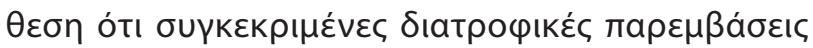

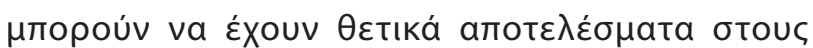

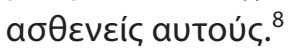

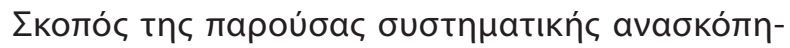

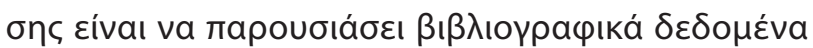

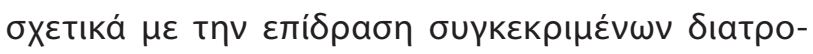

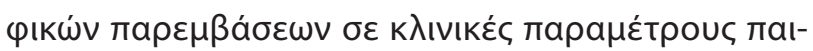

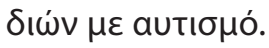

\section{MéӨodos}

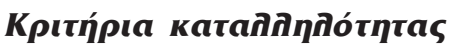

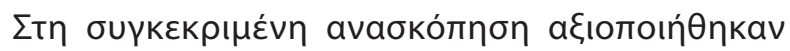

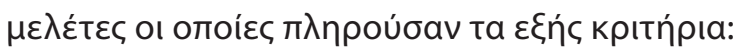

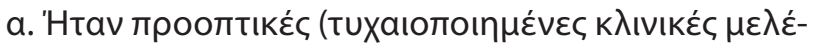

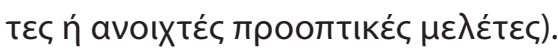

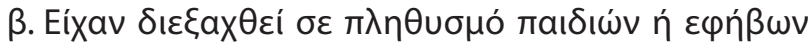

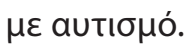

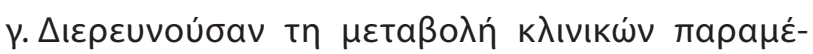

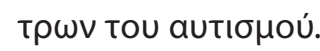

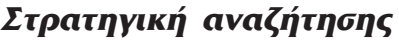

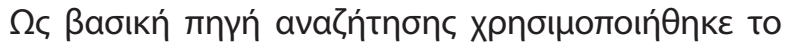

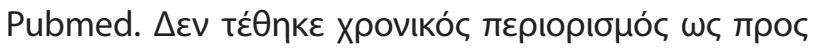

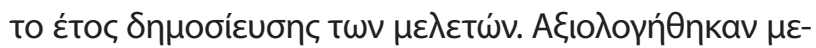

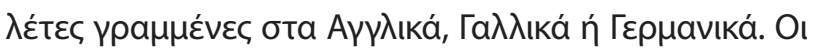

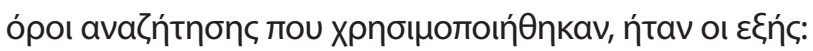
"autism", "vitamins", "minerals", "aminoacids", "diet", "carnitine", "probiotics", "prebiotics", "fattyacids", "nutritional interventions", "dietary interventions", "diets". 
А

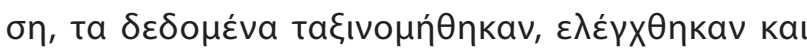

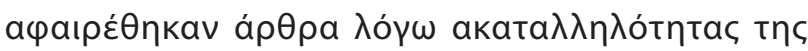

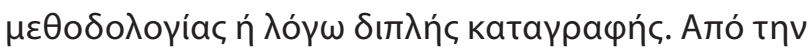

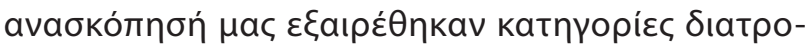

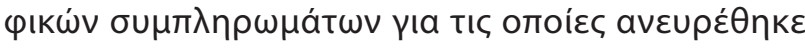

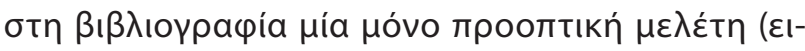
kóva 1).

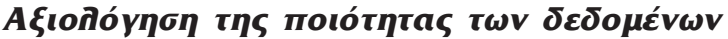

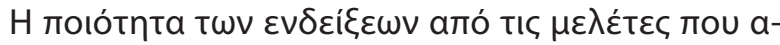

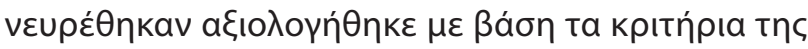

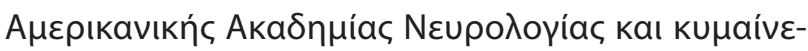

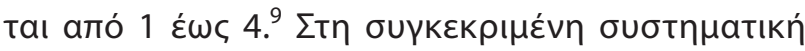

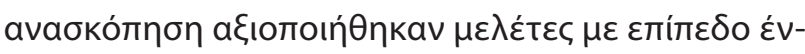

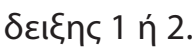

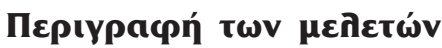

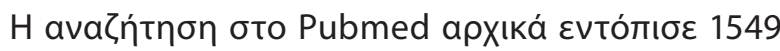

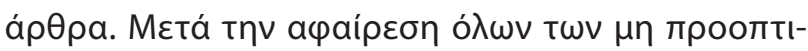

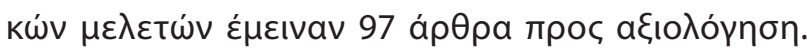

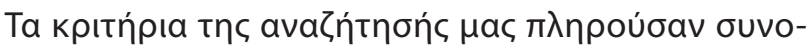

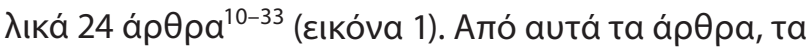

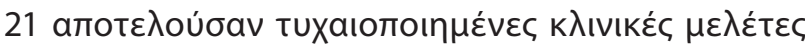

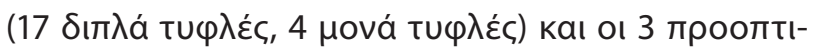

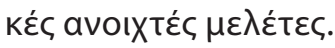

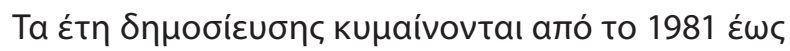

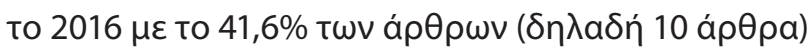

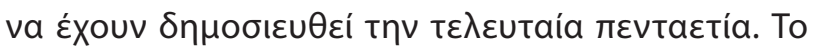

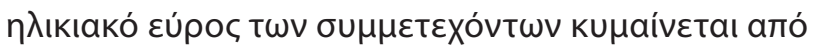

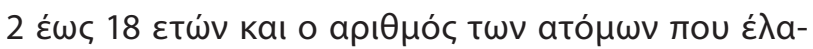

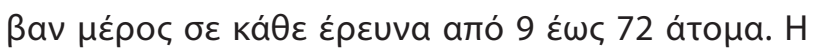

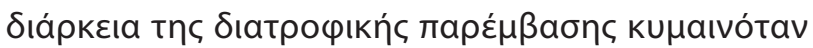

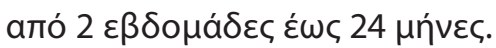

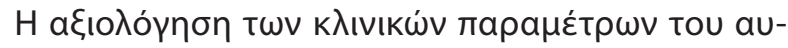

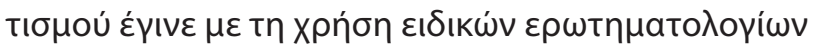

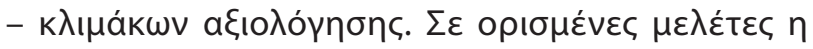

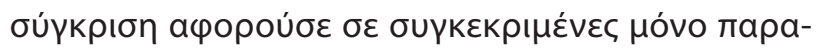

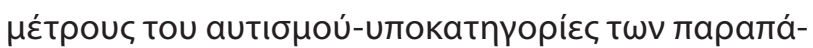

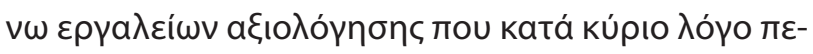

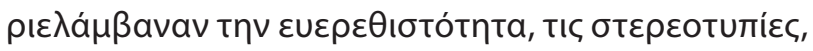

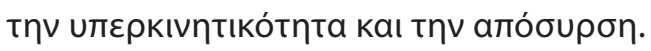

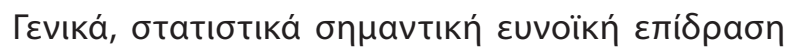

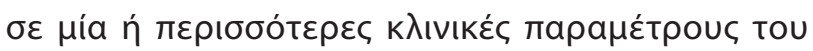

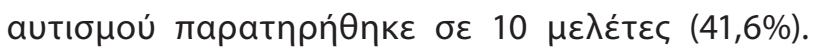

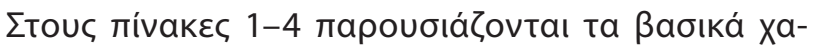

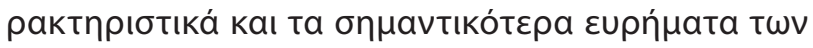

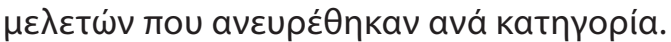

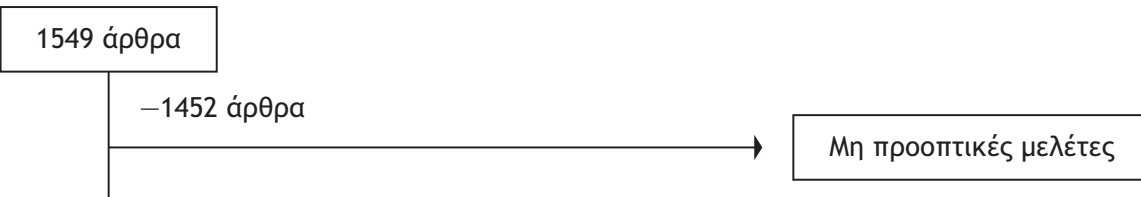

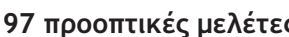

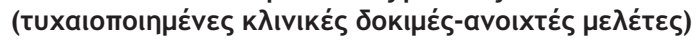

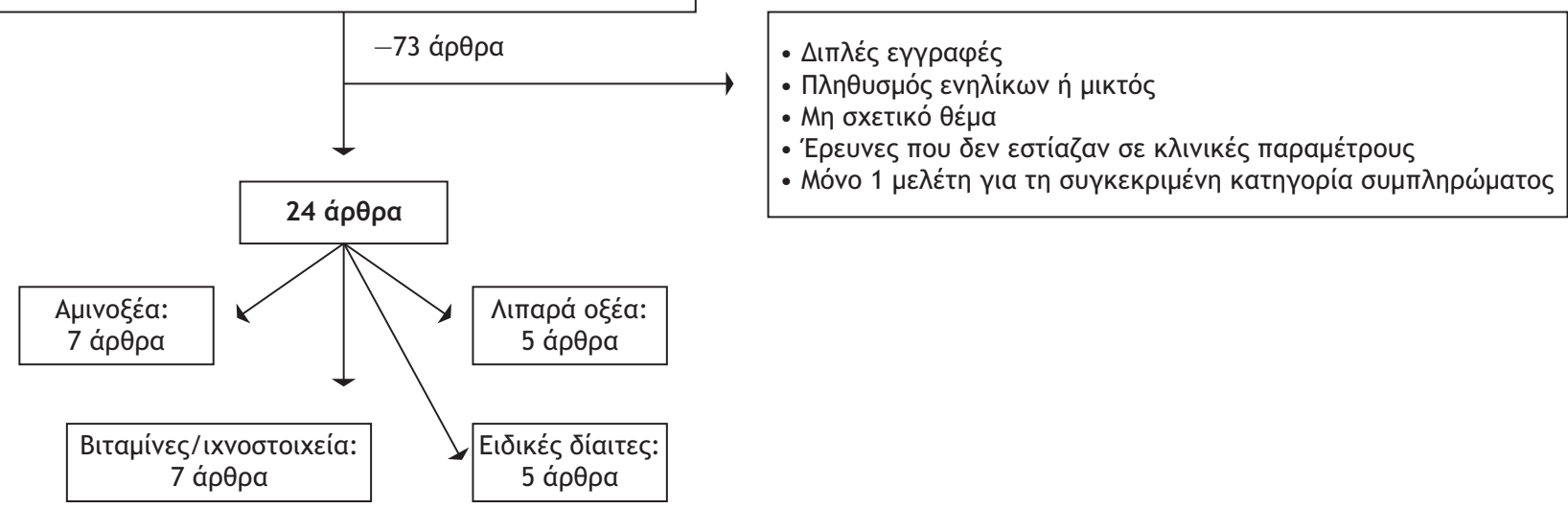

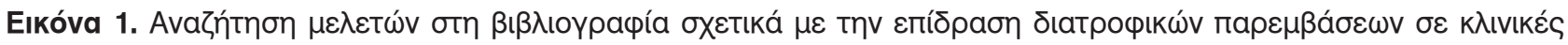

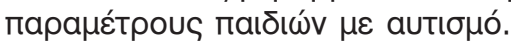




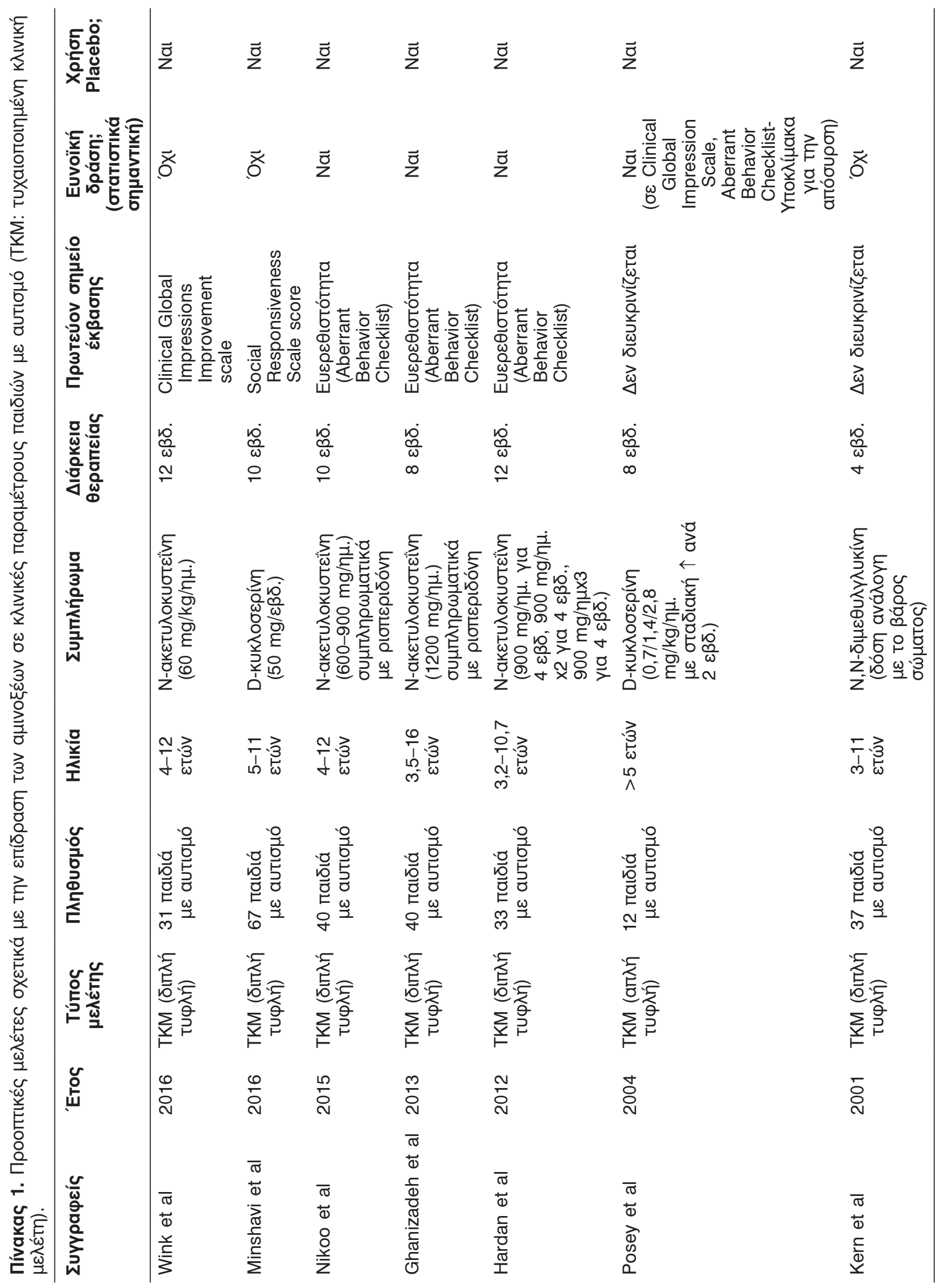




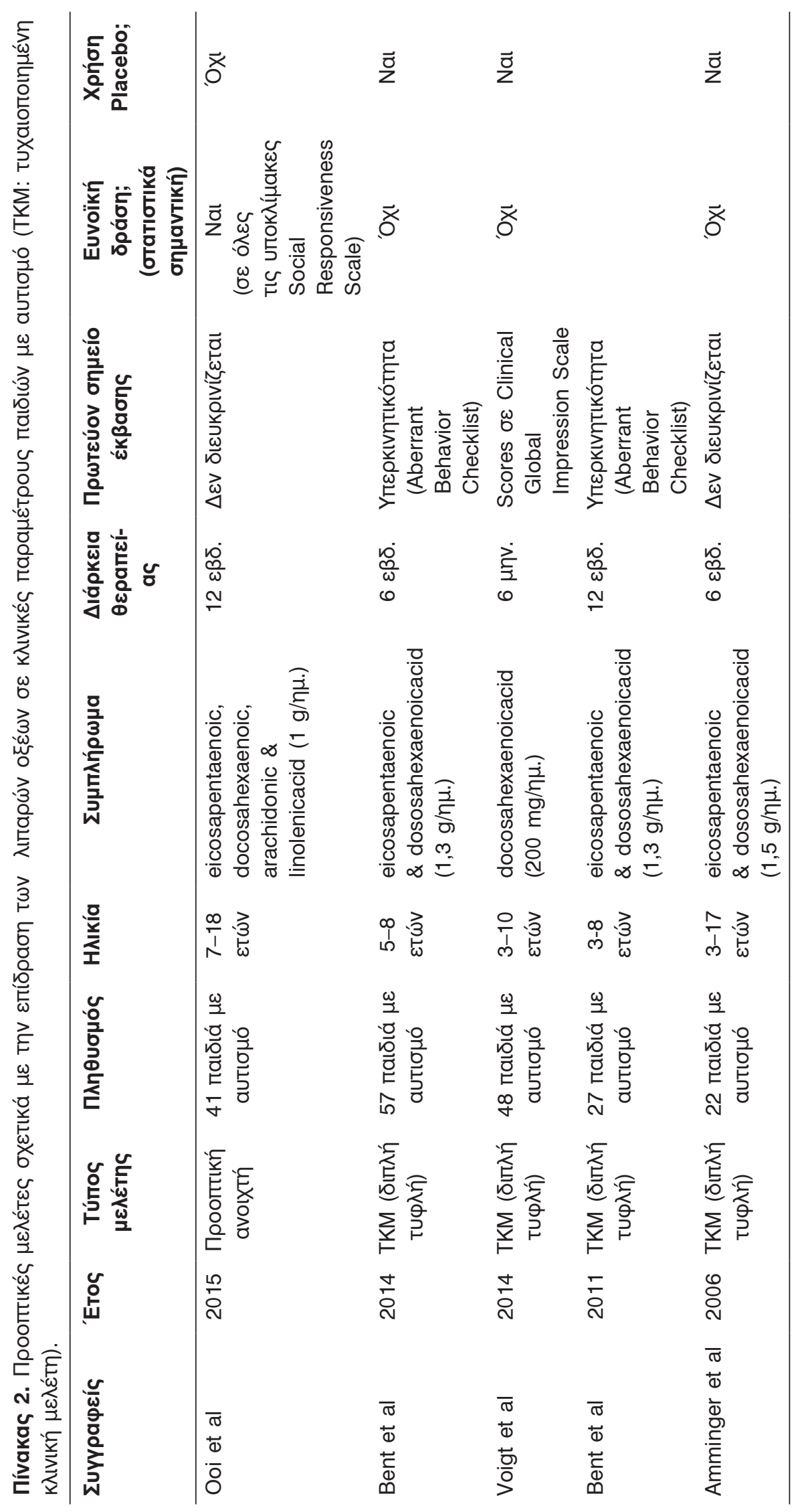




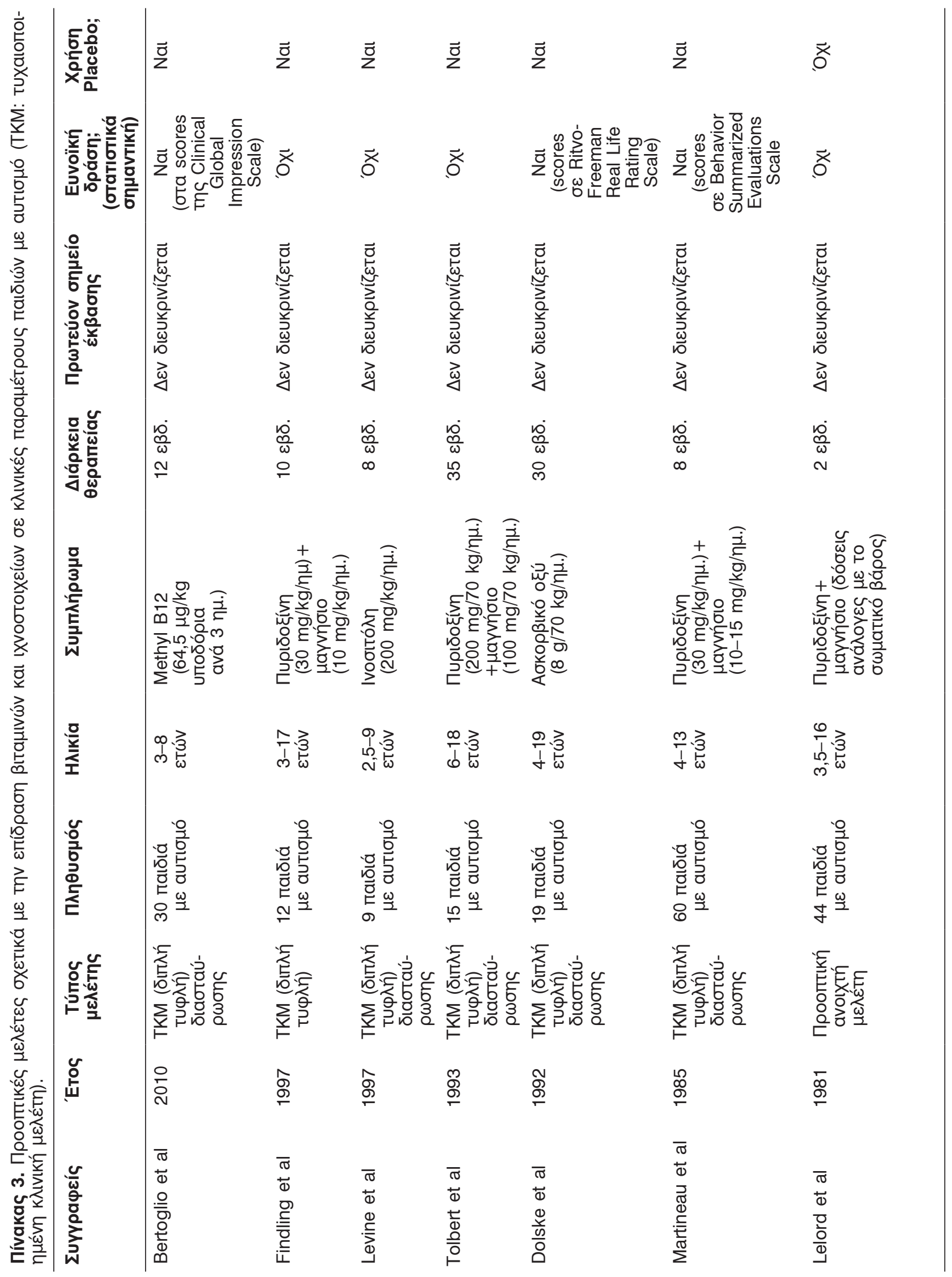




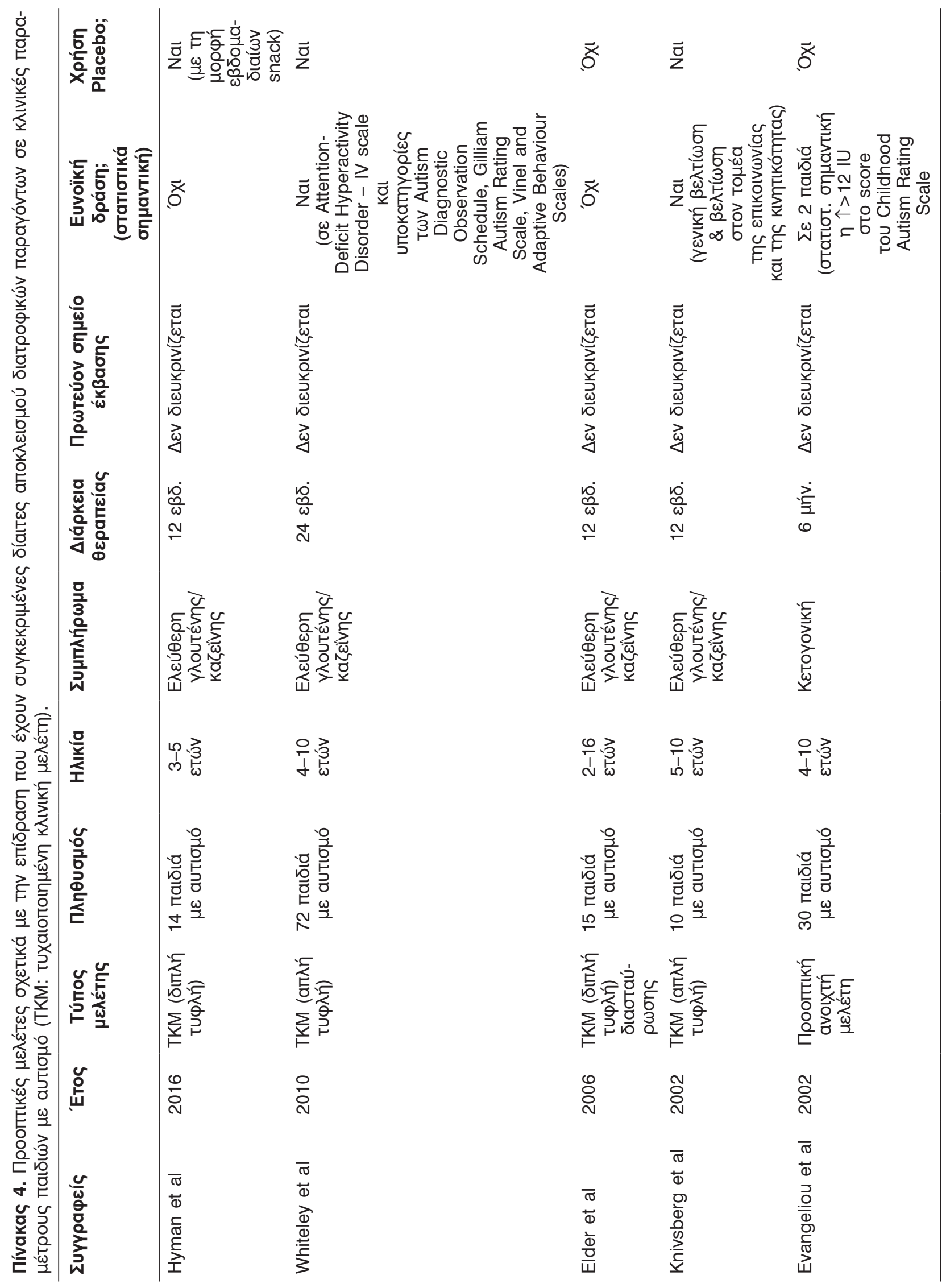




\section{Aptvoß́́a}

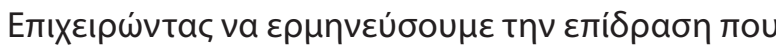

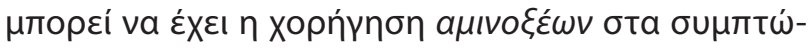

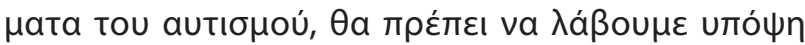

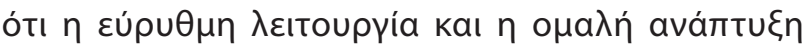

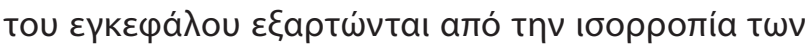

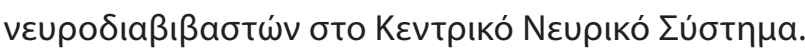

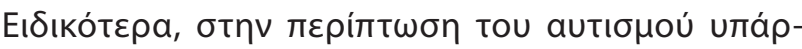

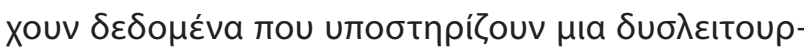

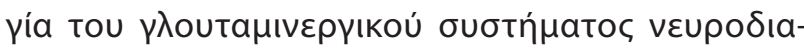

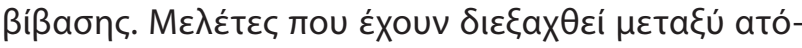

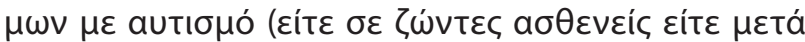

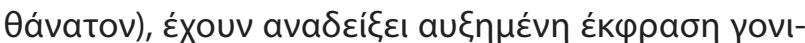

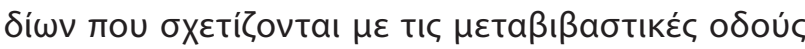

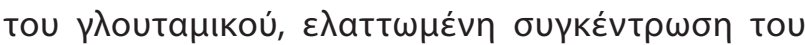

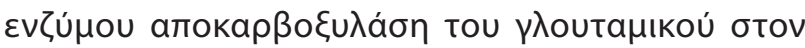

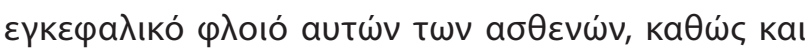

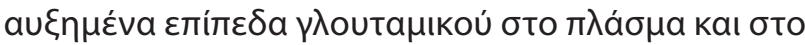

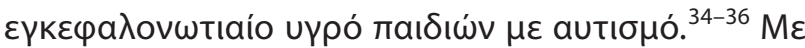

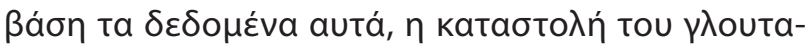

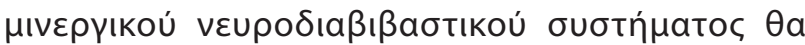

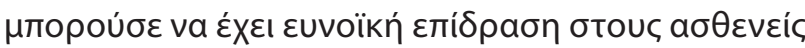

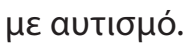

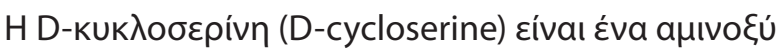

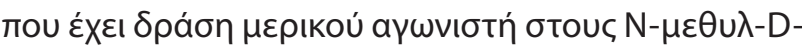

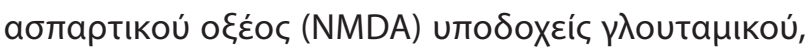

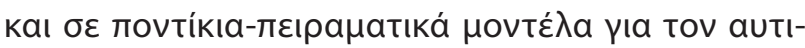

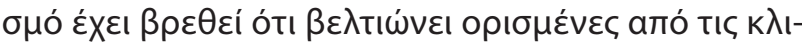

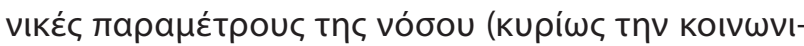

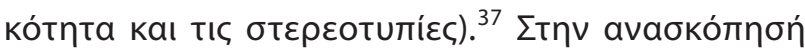

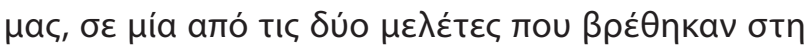

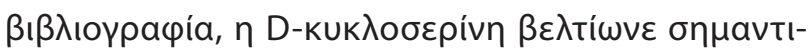

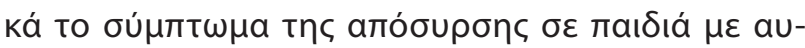
тıбнó.

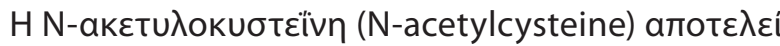

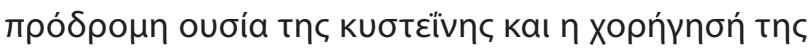

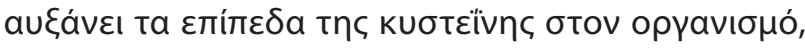

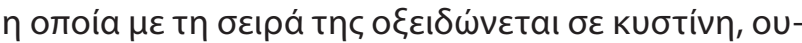

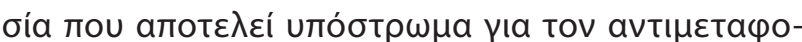

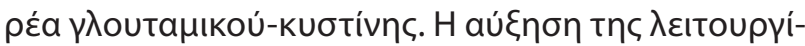

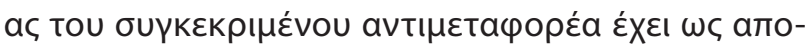

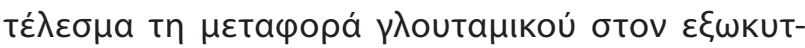

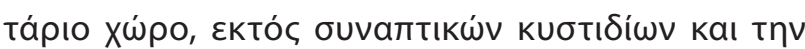

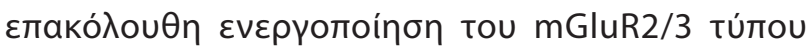

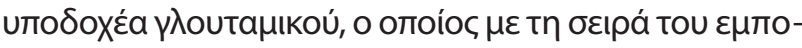

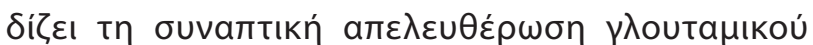

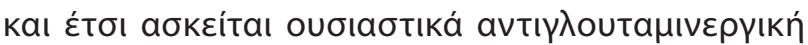

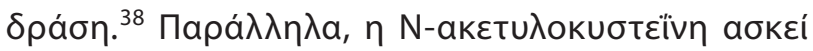

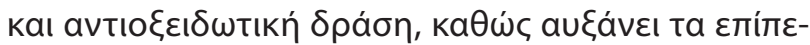

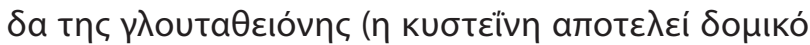

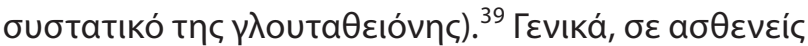

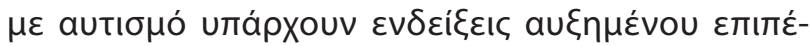

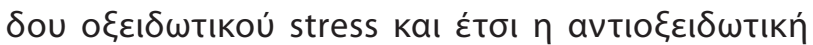

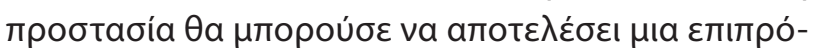

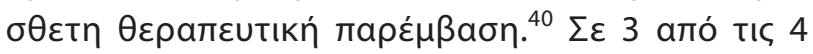

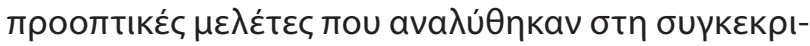

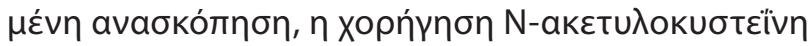

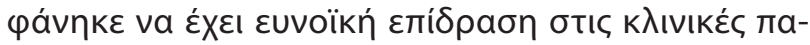

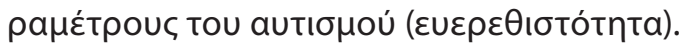

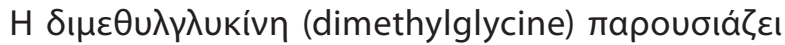

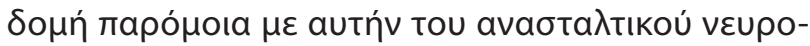

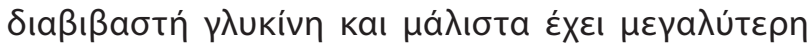

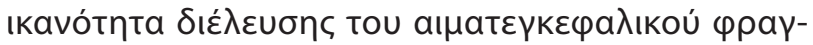

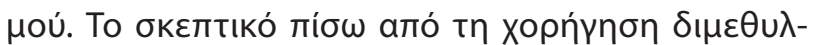

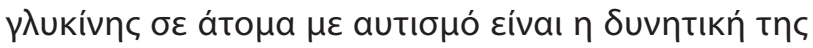

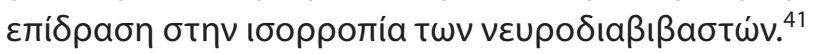

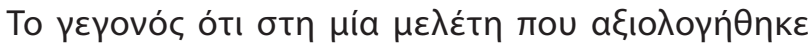

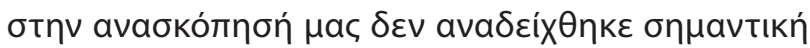

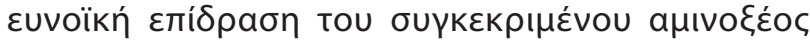

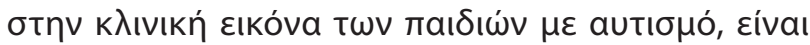

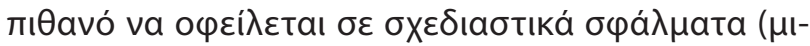

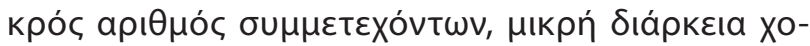

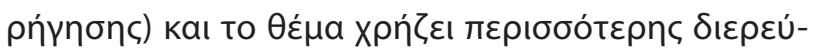

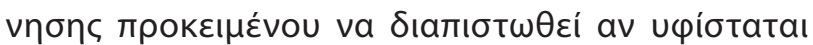

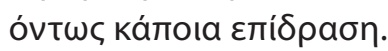

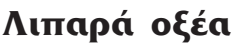

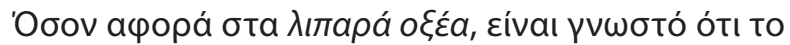

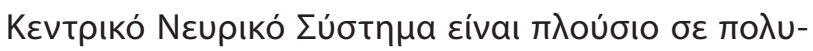

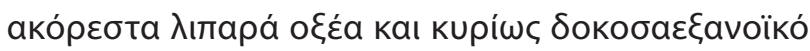

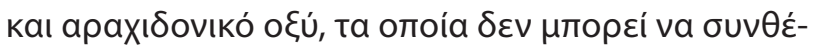

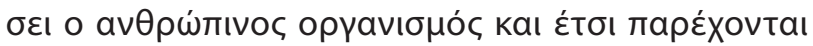

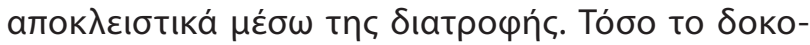

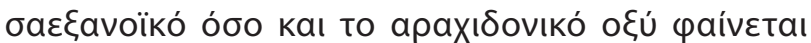

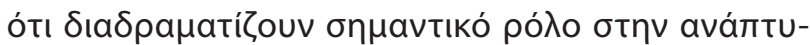

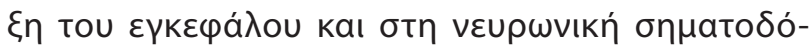

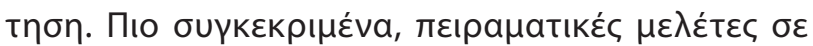

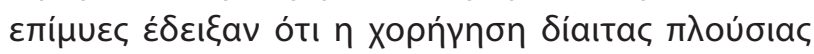

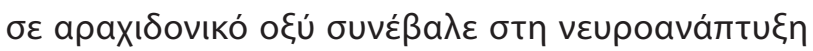

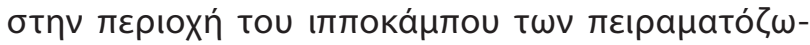

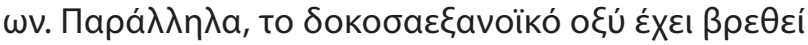




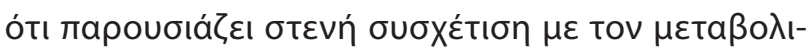

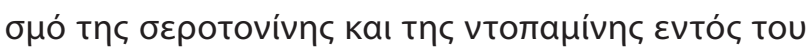

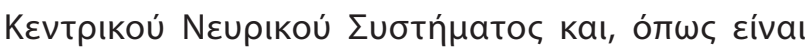

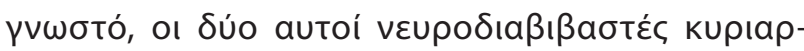

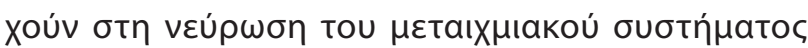

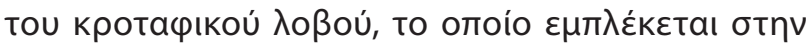

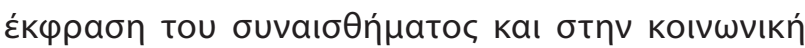
$a \lambda \lambda \eta \lambda \varepsilon \pi i \delta$ paon. ${ }^{42-44}$

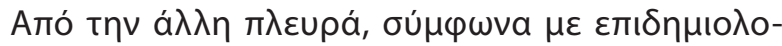

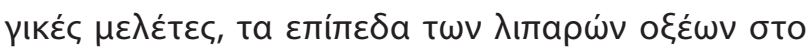

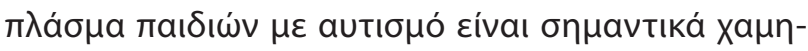

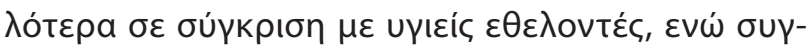

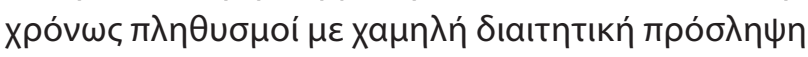

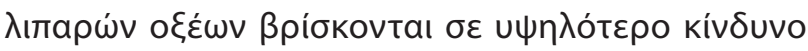

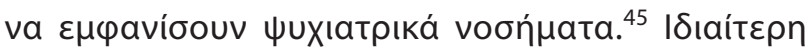

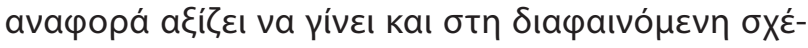

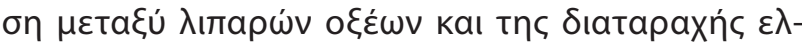

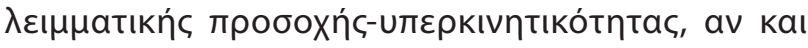

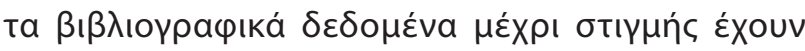

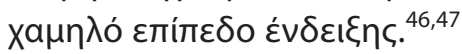

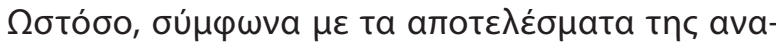

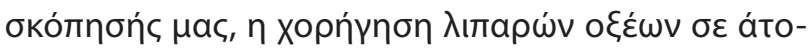

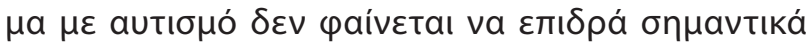

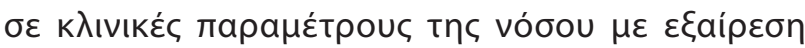

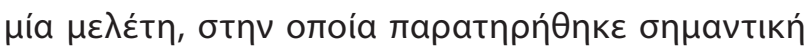

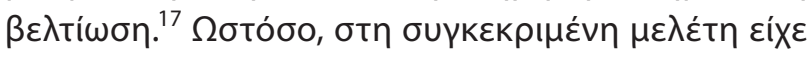

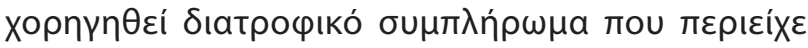

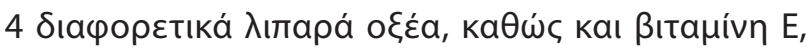

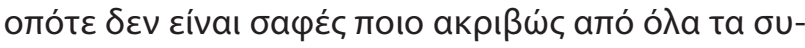

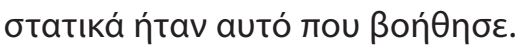

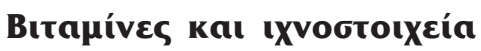

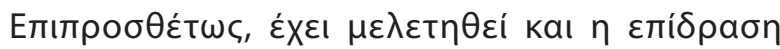

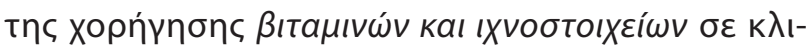

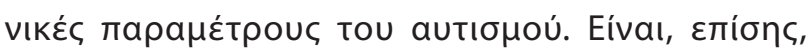

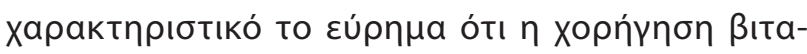

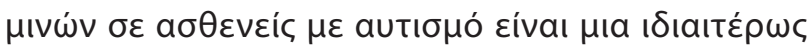

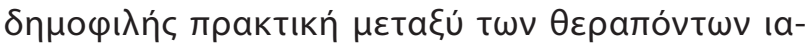

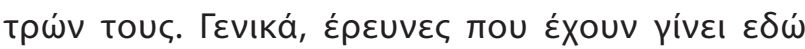

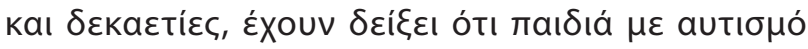

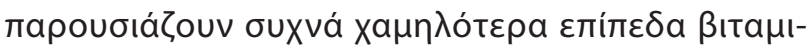

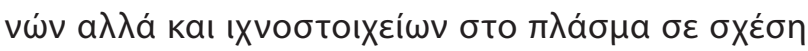

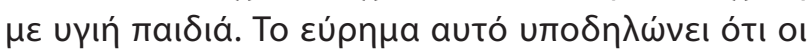

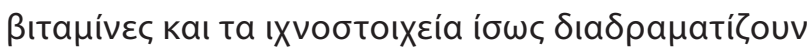

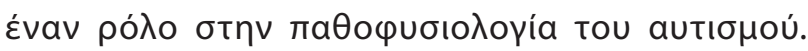

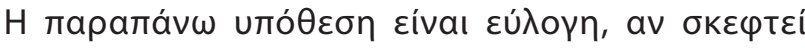

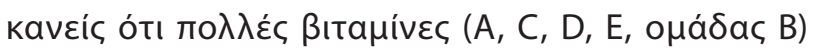
kaı ıxvootoıx

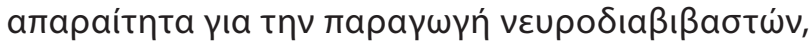

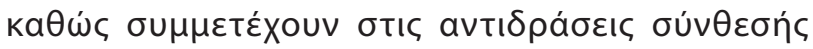

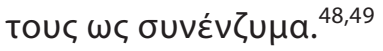

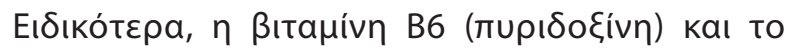

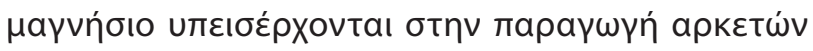

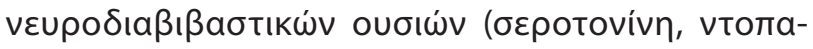

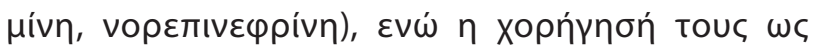

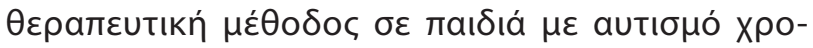

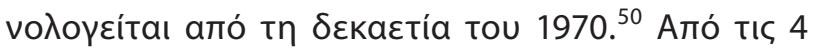

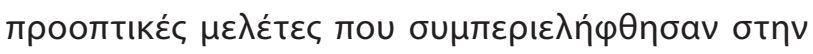

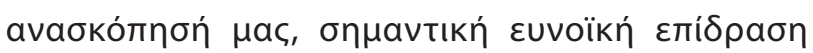

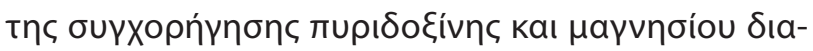

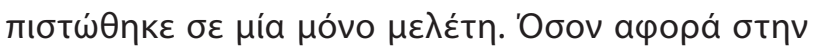

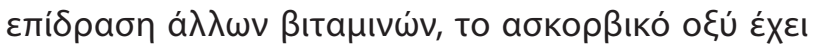

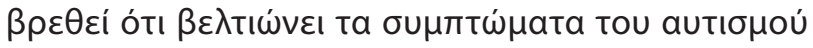

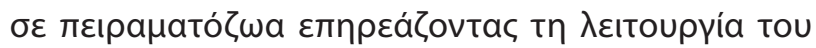

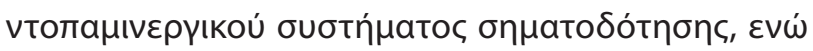

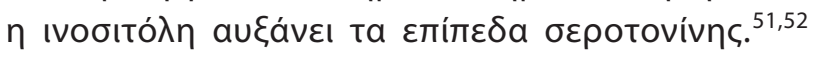

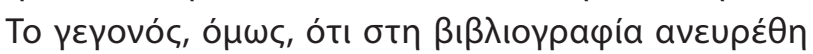

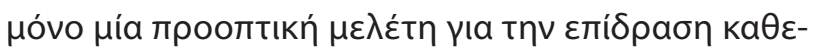

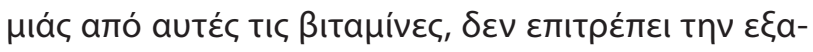

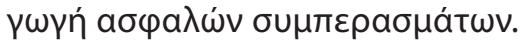

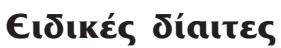

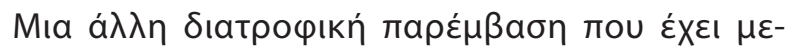

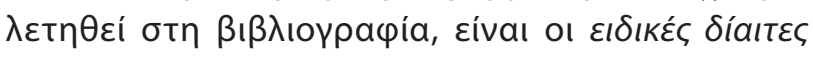

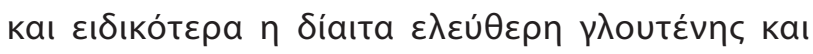

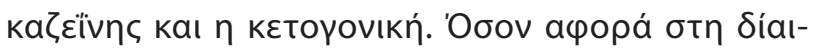

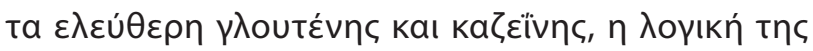

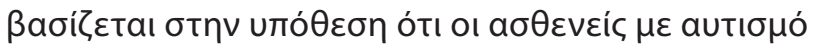

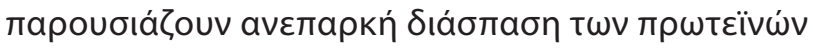

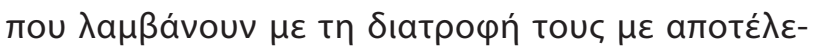

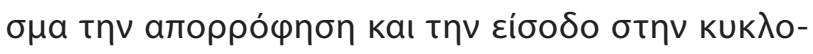

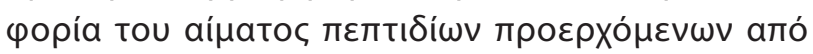

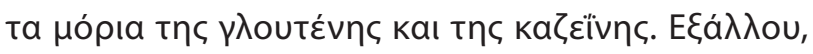

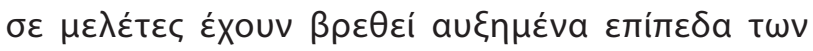

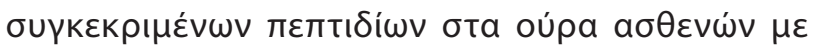

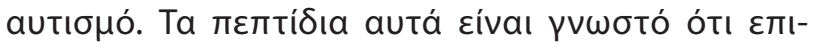

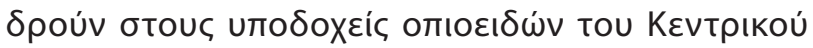

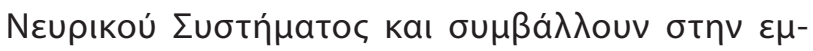

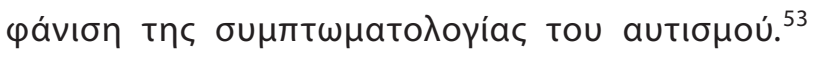

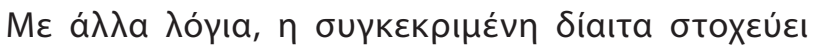

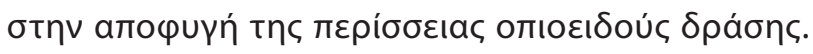

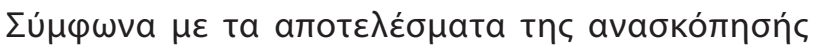




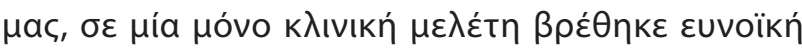

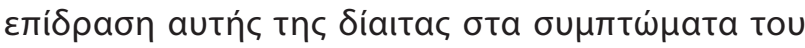

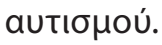

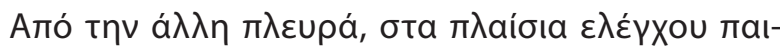

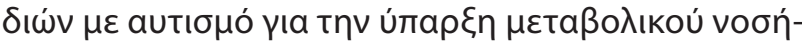

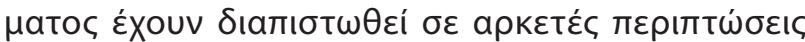

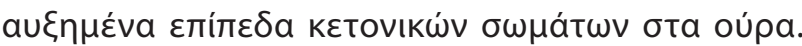

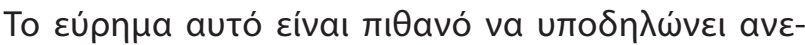

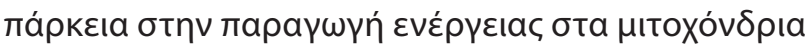

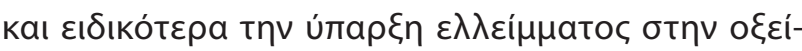

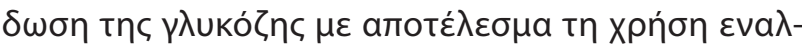

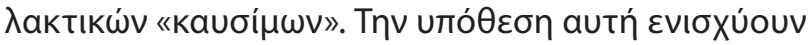

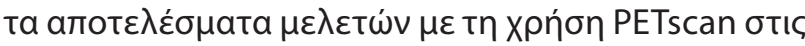

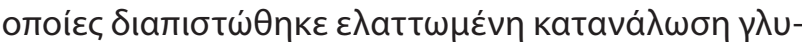

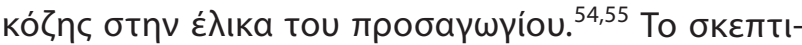

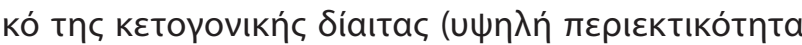

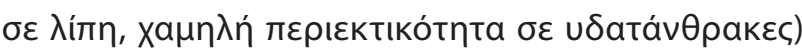

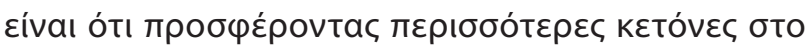

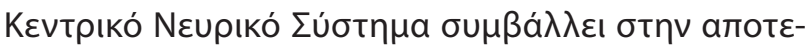

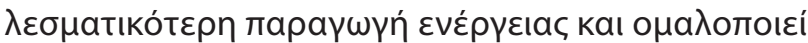

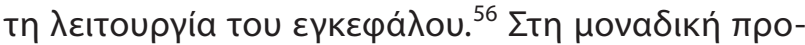

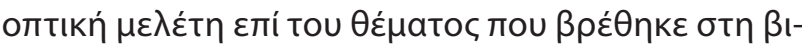

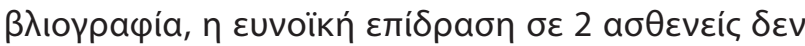

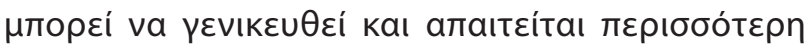

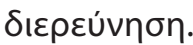

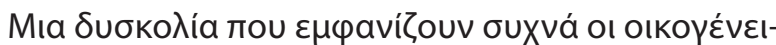

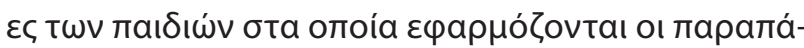

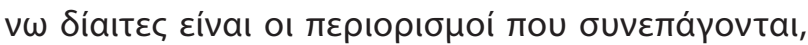

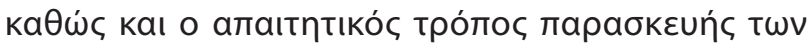

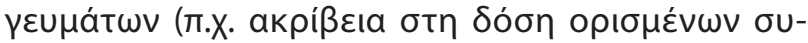

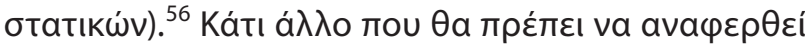

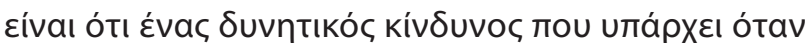

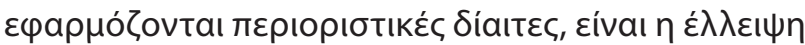

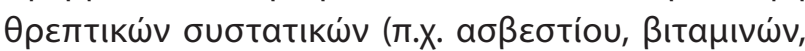

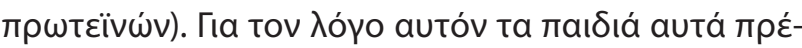

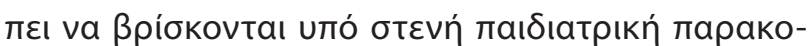

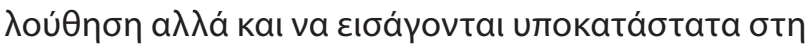

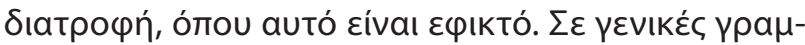

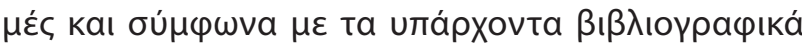

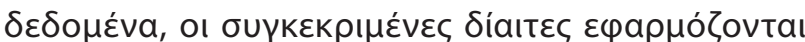

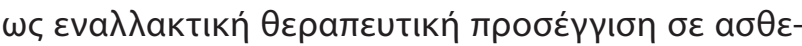

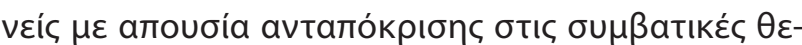

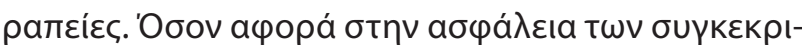

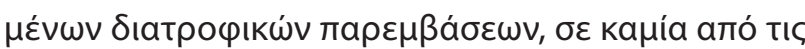

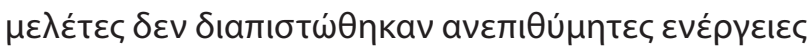

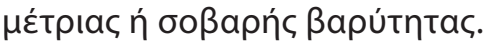

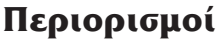

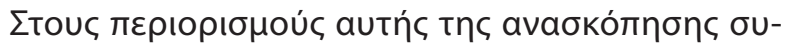

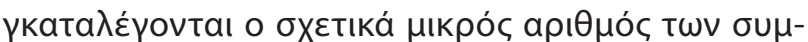

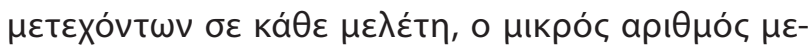

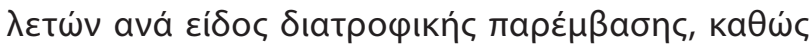

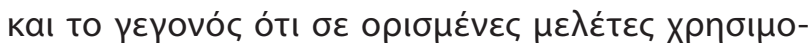

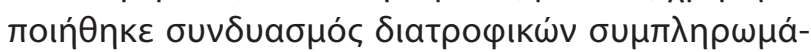

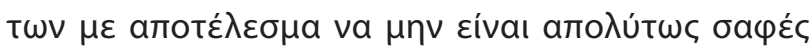

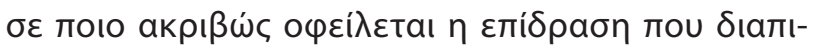

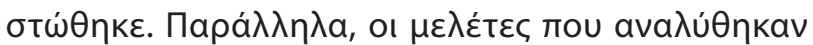

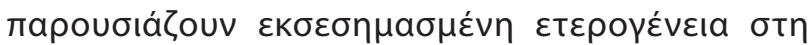

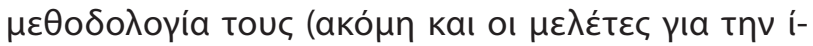

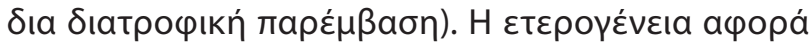

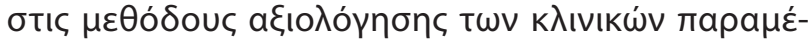

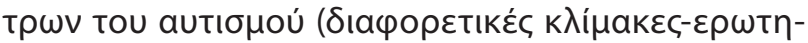

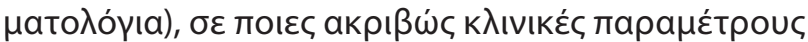

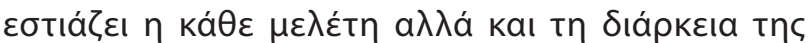

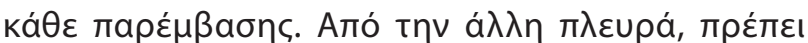

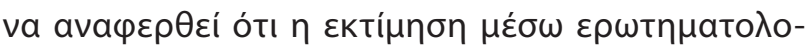

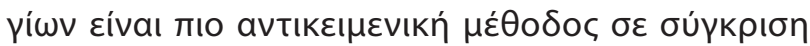

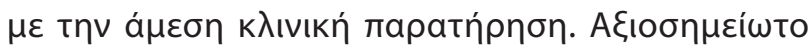

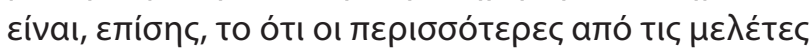

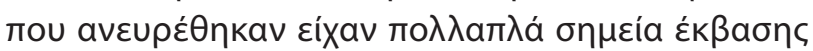

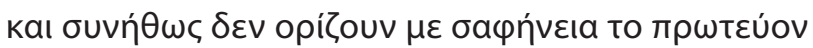

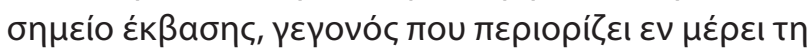

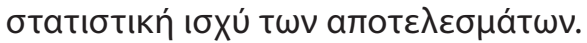

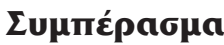

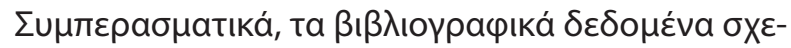

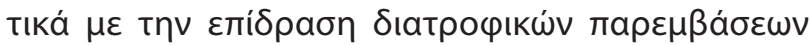

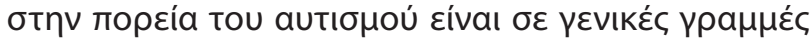

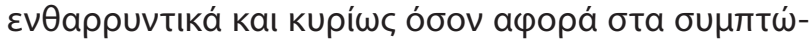

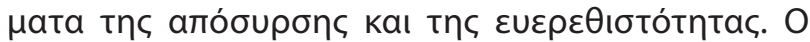

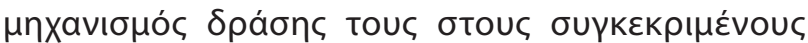

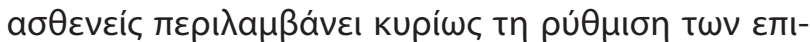

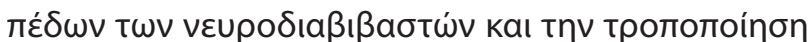

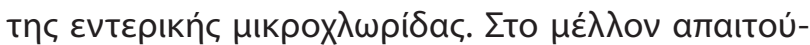

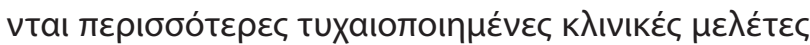

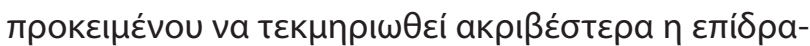

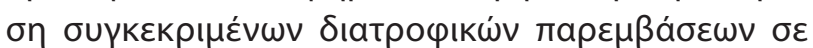

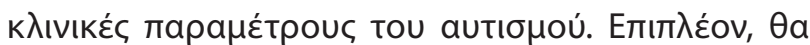

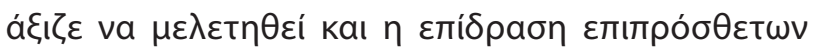

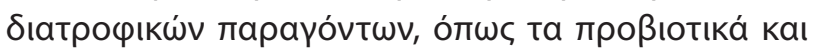

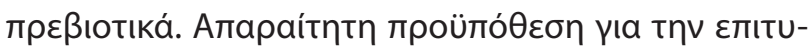

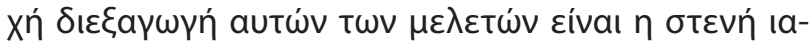

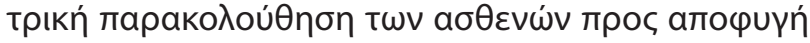

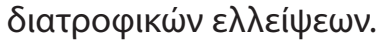




\title{
Is there place for nutrition in the treatment of children with autism spectrum disorder?
}

\author{
M. Gogou, ${ }^{1}$ G. Kolios ${ }^{2}$ \\ '2nd Department of Pediatrics, University General Hospital AHEPA, Thessaloniki, \\ ${ }^{2}$ Laboratory of Pharmacology, Medical School of Democritus University of Thrace, Alexandroupolis, Greece
}

Psychiatriki 2020, 31:57-69

\begin{abstract}
Autism is a neurodevelopmental disorder associated with significant social and financial burden. In recent years there has been an increasing interest in the use of dietary interventions as a complementary therapeutic option for these patients. The aim of this systematic review is to provide literature data about the effect of specific dietary interventions on clinical aspects of children with autism. For this reason, a literature search was conducted using Pubmed as the medical database source. No year-of-publication restriction was placed. Prospective studies conducted in pediatric populations and evaluating changes in clinical aspects of autism were considered. Types of dietary interventions evaluated in these studies included amino acids, fatty acids, vitamins/minerals, as well as specific diets (free of gluten/casein, ketogenic). The underlying mechanism of action of nutritional interventions in this pediatric population mainly includes regulation of neurotransmitters levels, as well as modification of gut microbiota. More specifically, $\mathrm{N}$-acetylcysteine was shown to exert a beneficial effect on symptoms of irritability. This beneficial effect could be attributed to its antiglutamergic and antioxidative properties. With regards to fatty acids, it is known that they are involved in dopamine and serotonin metabolism, while low values of fatty acids have been reported in serum of patients with various neuropsychiatric disorders. However, their administration in children with autism did not make any difference in terms of clinical aspects of the disease. On the other hand, available literature data about effect of $D$-cycloserine, dimethylglycine and vitamins/minerals was either few or controversial. In parallel, we were able to identify in literature clinical studies showing a beneficial effect of gluten/casein-free and ketogenic diet on clinical phenotype of autism. Finally, it should be highlighted that no moderate or serious adverse events were reported in any of the above nutritional interventions. In general, current literature data is encouraging. Nevertheless, more randomized clinical trials are needed to more clearly confirm the effect of specific dietary interventions on clinical aspects of autism.
\end{abstract}

Key words: Autism, amino acids, fatty acids, vitamins, therapeutic diets.

\section{Bıßntoypacpía}

1. Myers SM, Johnson CP; American Academy of Pediatrics Council on Children With Disabilities. Management of children with autism spectrum disorders. Pediatrics 2007, 120:11621182, doi: 10.1542/peds.2007-2362

2. Global Burden of Disease Study 2013 Collaborators. Global, regional, and national incidence, prevalence, and years lived with disability for 301 acute and chronic diseases and injuries in 188 countries, 1990-2013: a systematic analysis for the Global Burden of Disease Study 2013. Lancet 2015, 386:743-800, doi: $10.1016 /$ S0140-6736(15)60692-4
3. Center for Disease Control and Protection. Data \& Statistics on Autism Spectrum Disorder. Available from http://www.cdc.gov/ ncbddd/autism/index.html

4. Lightdale JR. The Gut Speaks: Reframing the Role of Pediatric Gastroenterologists Caring for Children With Autism and Gastrointestinal Symptoms. J Pediatr Gastroenterol Nutr 2016,63:313-314, doi: 10.1097/MPG.0000000000001319

5. Finegold SM, Molitoris D, Song Y, Liu C, Vaisanen ML, Bolte $E$ et al. Gastrointestinal microflora studies in late-onset autism. Clin Infect Dis 2002, 35:6-16, doi: 10.1086/341914 
6. Adams JB, Johansen LJ, Powell LD, Quig D, Rubin RA. Gastrointestinal flora and gastrointestinal status in children with autism--comparisons to typical children and correlation with autism severity. BMC Gastroenterol 2011, 11:22, doi: 10.1186/1471-230X-11-22

7. Francis K. Autism interventions: a critical update. Dev Med Child Neurol 2005, 47:493-499, doi: 10.1017/s0012162205000952

8. Grossi E, Melli S, Dunca D, Terruzzi V. Unexpected improvement in core autism spectrum disorder symptoms after longterm treatment with probiotics. SAGE Open Med Case Rep 2016, 4:2050313X16666231, doi: 10.1177/2050313X16666231

9. Neurology. The official journal of the American Academy of Neurology. Available from http://www.neurology.org/site/misc/ NeurologyFiller.pdf

10. Wink LK, Adams R, Wang Z, Klaunig JE, Plawecki MH, Posey DJ et al. A randomized placebo-controlled pilot study of $\mathrm{N}$-acetylcysteine in youth with autism spectrum disorder. $\mathrm{Mol}$ Autism 2016, 7:26, doi: 10.1186/s13229-016-0088-6

11. Minshawi NF, Wink LK, Shaffer R, Plawecki MH, Posey DJ, Liu H et al. A randomized, placebo-controlled trial of $\mathrm{D}$-cycloserine for the enhancement of social skills training in autism spectrum disorders. Mol Autism 2016, 7:2, doi: 10.1186/s13229-015-0062-8

12. Nikoo M, Radnia H, Farokhnia M, Mohammadi MR, Akhondzadeh S.N-acetylcysteine as an Adjunctive Therapy to Risperidone for Treatment of Irritability in Autism: A Randomized, DoubleBlind, Placebo-Controlled Clinical Trial of Efficacy and Safety. Clin Neuropharmacol 2015, 38:11-17, doi: 10.1097/ WNF.0000000000000063

13. Ghanizadeh A, Moghimi-Sarani E. A randomized double blind placebo controlled clinical trial of $\mathrm{N}$-acetylcysteine added to risperidone for treating autistic disorders. BMC Psychiatry 2013, 13:196, doi: 10.1186/1471-244X-13-196

14. Hardan AY, Fung LK, Libove RA, Obukhanych TV, Nair S, Herzenberg LA et al. A Randomized Controlled Pilot Trial of Oral N-acetylcysteine in Children with Autism. Biol Psychiatry 2012, 71:956-961, doi: 10.1016/j.biopsych.2012.01.014

15. Posey DJ, Kem DL, Swiezy NB, Sweeten TL, Wiegand RE, McDougle CJ. A Pilot Study of D-Cycloserine in Subjects With Autistic Disorder. Am J Psychiatry 2004, 161:2115-2117, doi: 10.1176/appi.ajp.161.11.2115

16. Kern JK, Miller VS, Cauller PL, Kendall PR, Mehta PJ, Dodd M Effectiveness of N,N-Dimethylglycine in Autism and Pervasive Developmental Disorder. J Child Neurol 2001, 16:169-173, doi: 10.1177/088307380101600303

17. Ooi YP, Weng SJ, Jang LY, Low L, Seah J, Teo S et al. Omega-3 fatty acids in the management of autism spectrum disorders: findings from an open-label pilot study in Singapore. Eur J Clin Nutr 2015, 69:969-971, doi: 10.1038/ejcn.2015.28

18. Bent S, Hendren RL, Zandi T, Law K, Choi JE, Widjaja F et al. Internet-Based, Randomized Controlled Trial of Omega-3 Fatty Acids for Hyperactivity in Autism. J Am Acad Child Adolesc Psychiatry 2014, 53:658-666, doi: 10.1016/j.jaac.2014.01.018

19. Voigt RG, Mellon MW, Katusic SK, Weaver AL, Matern D, Mellon $B$ et al. Dietary Docosahexaenoic Acid Supplementation in Children With Autism. J Pediatr Gastroenterol Nutr 2014, 58:715722, doi: 10.1097/MPG.0000000000000260
20. Bent S, Bertoglio K, Ashwood P, Bostrom A, Hendren RL. A Pilot Randomized Controlled Trial of Omega-3 Fatty Acids for Autism Spectrum Disorder. J Autism Dev Disord 2011, 41:545-554, doi: 10.1007/s10803-010-1078-8

21. Amminger GP, Berger GE, Schäfer MR, Klier C, Friedrich MH, Feucht M. Omega-3 Fatty Acids Supplementation in Children with Autism: A Double-blind Randomized, Placebo-controlled Pilot Study. Biol Psychiatry 2007, 61:551-553, doi: 10.1016/j. biopsych.2006.05.007

22. Bertoglio K, Jill James S, Deprey L, Brule N, Hendren RL. Pilot study of the effect of methyl B12 treatment on behavioral and biomarker measures in children with autism. $J$ Altern Complement Med 2010, 16:555-560, doi: 10.1089/acm.2009. 0177

23. Findling RL, Maxwell K, Scotese-Wojtila L, Huang J, Yamashita T, Wiznitzer M. High-Dose Pyridoxine and Magnesium Administration in Children with Autistic Disorder: An Absence of Salutary Effects in a Double-Blind, PlaceboControlled Study. J Autism Dev Disord 1997, 27:467-478, doi: 10.1023/a:1025861522935

24. Levine J, Aviram A, Holan A, Ring A, Barak Y, Belmaker RH. Inositol treatment of autism. J Neural Transm (Vienna) 1997, 104:307-310, doi: 10.1007/s007020050200

25. Tolbert L, Haigler T, Waits MM, Dennis T. Brief Report: Lack of Response in an Autistic Population to a Low Dose Clinical Trial of Pyridoxine Plus Magnesium. J Autism Dev Disord 1993, 23:193-199, doi: 10.1007/bf01066428

26. Dolske MC, Spollen J, McKay S, Lancashire E, Tolbert L. A preliminary trial of ascorbic acid as supplemental therapy for autism. Prog Neuropsychopharmacol Biol Psychiatry 1993, 17:765-774, doi: 10.1016/0278-5846(93)90058-z

27. Martineau J, Barthelemy C, Garreau B, Lelord G. Vitamin B6, magnesium, and combined B6-Mg: therapeutic effects in childhood autism. Biol Psychiatry 1985, 20:467-478, doi: 10.1016/0006-3223(85)90019-8

28. Lelord G, Muh JP, Barthelemy C, Martineau J, Garreau B, Callaway E. Effects of Pyridoxine and Magnesium on AutisticSymptoms--Initial Observations. J Autism Dev Disord 1981, 11:219-230, doi: 10.1007/bf01531686

29. Hyman SL, Stewart PA, Foley J, Cain U, Peck R, Morris DD et al. The Gluten-Free/Casein-Free Diet: A Double-Blind Challenge Trial in Children with Autism. J Autism Dev Disord 2016, 46:205220, doi: 10.1007/s10803-015-2564-9

30. Whiteley P, Haracopos D, Knivsberg AM, Reichelt KL, Parlar S, Jacobsen $\mathrm{J}$ et al. The ScanBritrandomised, controlled, singleblind study of a gluten- and casein-free dietary intervention for children with autism spectrum disorders. Nutr Neurosci 2010, 13:87-100, doi: 10.1179/147683010X12611460763922

31. Elder JH, Shankar M, Shuster J, Theriaque D, Burns S, Sherrill L. The gluten-free, casein-free diet in autism: results of a preliminary double blind clinical trial. J Autism Dev Disord 2006, 36:413-420, doi: 10.1007/s10803-006-0079-0

32. Evangeliou A, Vlachonikolis I, Mihailidou H, Spilioti M, Skarpalezou A, Makaronas $\mathrm{N}$ et al. Application of a ketogenic diet in children with autistic behavior: pilot study. J Child Neurol 2003, 18:113-118, doi: 10.1177/08830738030180020501 
33. Knivsberg AM, Reichelt KL, Høien T, Nødland M. A Randomised, controlled study of dietary intervention in autistic syndromes. Nutr Neurosci 2002, 5:251-261, doi: 10.1080/10284150290028945

34. Purcell A, Jeon O, Zimmerman A, Blue ME, Pevsner J. Postmortem brain abnormalities of the glutamate neurotransmitter system in autism. Neurology 2001, 57:1618-1628, doi: 10.1212/wnl.57.9.1618

35. Fatemi SH, Halt AR, Stary JM, Kanodia R, Schulz SC, Realmuto GR. Glutamic acid decarboxylase 65 and $67 \mathrm{kDa}$ proteins are reduced in autistic parietal and cerebellar cortices. Biol Psychiatry 2012, 52:805-810, doi: 10.1016/s00063223(02)01430-0

36. Shinohe A, Hashimoto K, Nakamura K, Tsujii M, Iwata Y, Tsuchiya $\mathrm{KJ}$ et al. Increased serum levels of glutamate in adult patients with autism. Prog Neuropsychopharmacol Biol Psychiatry 2006, 30:1472-1477, doi: 10.1016/j.pnpbp.2006.06.013

37. Modi ME, Young LJ. D-cycloserine facilitates socially reinforced learning in an animal model relevant to autism spectrum disorders. Biol Psychiatry 2011, 70:298-304, doi: 10.1016/j. biopsych.2011.01.026

38. Baker DA, Xi ZX, Shen H, Swanson CJ, Kalivas PW. The origin and neuronal function of in vivo nonsynaptic glutamate. $J$ Neurosci 2002, 22:9134-9141, PMID: 12388621

39. Ghanizadeh A, Akhondzadeh S, Hormozi M, Makarem A, Abotorabi-Zarchi M, Firoozabadi A. Glutathione-related factors and oxidative stress in autism, a review. Curr Med Chem 2012, 19:4000-4005, doi: 10.2174/092986712802002572

40. Damodaran LPM, Arumugam G. Urinary oxidative stress markers in children with autism. Redox Rep 2011, 16:216-222, doi: 10.1179/1351000211Y.0000000012

41. Livine S, Myhre G, Smith G, Bums J. Effect of nutritional supplement containing $\mathrm{N}, \mathrm{N}$-dimethylglycine (DMG) on the racing standard bred. Equine Pract 1982, 4:16-18

42. Wainwright PE. Dietary essential fatty acids and brain function: a developmental perspective on mechanisms. Proc Nutr Soc 2002, 61:61-69, doi:10.1079/pns2001130

43. Bazan NG. Lipid signalling in neural plasticity, brain repair, and neuroprotection. Mol Neurobiol 2005, 32:89-103, doi: 10.1385/ $\mathrm{MN}: 32: 1: 089$

44. Hibbeln JR, Umhau JC, Linnoila M, George DT, Ragan PW, Shoaf SE et al. A replication study of violent and nonviolent subjects: cerebrospinal fluid metabolites of serotonin and dopamine are predicted by plasma essential fatty acids. Biol Psychiatry 1998, 44:243-249, doi: 10.1016/s0006-3223(98)00143-7

45. Bell JG, MacKinlay EE, Dick JR, MacDonald DJ, Boyle RM, Glen AC. Essential fatty acids and phospholipase A2 in autistic spectrum disorders. Prostaglandins Leukot Essent Fatty Acids 2004, 71:201-204, doi: 10.1016/j.plefa.2004.03.008

46. Noaghiul S, Hibbeln JR. Cross-national comparisons of seafood consumption and rates of bipolar disorders. Am J Psychiatry 2003, 160:2222-2227, doi: 10.1176/appi.ajp.160.12.2222

47. Raz R, Gabis L. Essential fatty acids and attention-deficit-hyperactivity disorder: a systematic review. Dev Med Child Neurol 2009, 51:580-592, doi: 10.1111/j.1469-8749.2009.03351.x

48. Adams JB, Audhya T, Vogelaar E. Nutritional abnormalities in autism and the effect of nutritional supplementation. Paper pre- sented at Conference of the National Autism Society of America, Pittsburgh, 16-20 July, 2003

49. Golnik AE, Ireland M. Complementary alternative medicine for children with autism: a physician survey. J Autism Dev Disord 2009, 39:996-1005, doi: 10.1007/s10803-009-0714-7

50. Coleman M, Steinberg G, Tippett J, Bhagavan HN, Coursin $\mathrm{DB}$, Gross $\mathrm{M}$ et al. A preliminary study of the effect of pyridoxine administration in a subgroup of hyperkinetic children: a double-blind crossover comparison with methylphenidate. Biol Psychiatry 1979, 14:741-751, PMID: 497303

51. Tolbert LC, Thomas TN, Middaugh LD, Zemp JW. Ascorbate blocks amphetamine-induced turning behavior in rats with unilateral nigro-striatal lesions. Brain Res Bull 1979, 4:43-48, doi: 10.1016/0361-9230(79)90056-x

52. Rahman S, Neuman RS. Myo-inositol reduces serotonin (5-HT2) receptor induced homologous and heterologous desensitization. Brain Res 1993, 631:349-351, doi: 10.1016/0006-8993 (93) $91557-9$

53. Whiteley P, Rodgers J, Savery D, Shattock P. A Gluten-Free Diet as an Intervention for Autism and Associated Spectrum Disorders: Preliminary Findings. Autism 1999, 3:45-65, doi: $10.1177 / 1362361399003001005$

54. Poggi-Travert $F$, Martin D, Billette de Villemeur T, Bonnefont JP, Vassault A, Rabier D et al. Metabolic intermediates in lactic acidosis: compounds, samples and interpretation. $J$ Inherit Metab Dis 1996, 19:478-488, doi: 10.1007/bf01799109

55. Haznedar MM, Buchsbaum MS, Wei TC, Hof PR, Cartwright C, Bienstock CA et al. Limbic circuitry in patients with autism spectrum disorders studied with positron emission tomography and magnetic resonance imaging. Am J Psychiatry 2000, 157:1994-2001, doi: 10.1176/appi.ajp.157.12.1994

56. Posey DJ, McDougle CJ. Pharmacotherapeutic management of autism. Expert Opin Pharmacother 2001, 2:587-600, doi: 10.1517/14656566.2.4.587

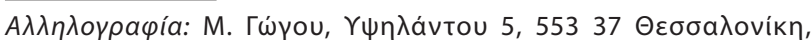

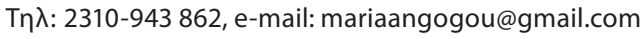

